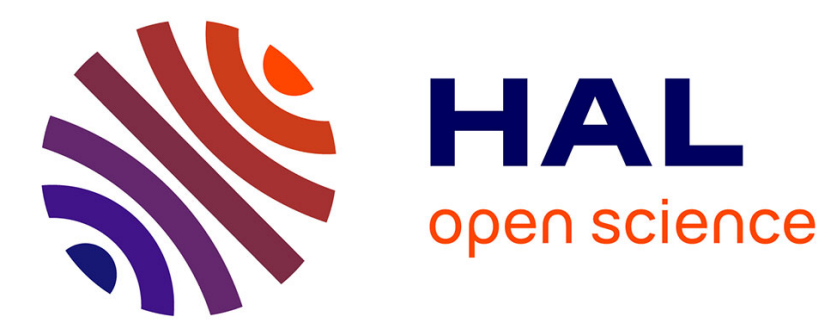

\title{
La référence à l'avenir en anglais contemporain : vers une énonciation médiatisée
}

\author{
Agnès Celle, Laure Lansari
}

\section{To cite this version:}

Agnès Celle, Laure Lansari. La référence à l'avenir en anglais contemporain: vers une énonciation médiatisée. Faits de langues, 2009, Le futur dans les langues, 33, pp.103-109. hal-01227122

HAL Id: hal-01227122

https://hal-univ-paris.archives-ouvertes.fr/hal-01227122

Submitted on 19 Feb 2021

HAL is a multi-disciplinary open access archive for the deposit and dissemination of scientific research documents, whether they are published or not. The documents may come from teaching and research institutions in France or abroad, or from public or private research centers.
L'archive ouverte pluridisciplinaire HAL, est destinée au dépôt et à la diffusion de documents scientifiques de niveau recherche, publiés ou non, émanant des établissements d'enseignement et de recherche français ou étrangers, des laboratoires publics ou privés. 


\title{
La référence à l'avenir en anglais contemporain : vers une énonciation médiatisée
}

\author{
Agnès Celle et Laure Lansari*
}

\section{INTRODUCTION}

On a tendance à résumer la référence à l'avenir en anglais à l'opposition entre deux modaux, shall et will. Pourtant, les études quantitatives qui ont été menées récemment (Berglund, 1997, 2000 ; Nesselhauf, 2007) montrent d'une part que shall est devenu résiduel dans cette fonction, d'autre part qu'il y a une nette augmentation des formes porteuses de l'aspect be-ing. D'après les corpus consultés, l'augmentation de be-ing est constante depuis le XVIIIème siècle dans toutes les variétés d'anglais. Le fait marquant du XXème siècle, c'est que be going to et will be -ing gagnent du terrain, et dans deux zones géographiques différentes. L'examen du corpus Archer et du corpus Brown (Celle \& Smith à paraître) montre en effet que will be-ing est la seule construction, parmi les différentes expressions faisant référence à l'avenir, dont l'emploi ait considérablement augmenté au cours du XXème siècle en anglais britannique. Entre 1961 et 1991 l'examen du LOB et du FLOB montre une augmentation de l'emploi de will be -ing de l'ordre de $53 \%$ contre $2,7 \%$ pour be going to. Il y a à l'heure actuelle plus de deux fois plus d'occurrences de will be-ing en anglais britannique qu'en anglais américain. Vice versa, c'est en anglais américain que be going to s'est le plus développé dans tous les registres, sous sa forme pleine et sous sa forme réduite gonna (voir Mair, 1997).

Nous voudrions montrer comment ces formes modifient la façon de référer à l'avenir du point de vue de l'intersubjectivité. Nous avançons l'hypothèse qu'un rapprochement est à faire entre ces différentes formes aspectuo-modales et l'énonciation médiatisée telle que l'a définie Guentchéva (1996:15-16). Cette

* Université Paris-Diderot Paris 7, CLILLAC-ARP et Université de Reims, CIRLEP. 
hypothèse accrédite l'idée que la référence à l'avenir passe par la modalité, à la différence de la référence au passé ou au présent. En d'autres termes, il n'y a pas de 'pure future' en anglais, et nous tenterons de montrer que même dans les emplois où, selon l'idée généralement admise, l'aspect neutralise la modalité, une forme d'énonciation médiate est en jeu, qui reste en rapport avec la modalité, à un autre titre.

\section{WILL : AJUSTEMENT A LA SITUATION}

La prédiction qu'exprime will sous sa forme pleine ou contractée ${ }^{2}$, met en jeu un rapport direct à la situation, et cela dans deux sens. Soit on prédit une occurrence future à partir des données de la situation, soit on explique la situation en vertu d'un principe plus général. Lorsqu'on prédit une occurrence future, celle-ci découle soit de la valeur radicale du modal, soit d'un raisonnement. Dans le premier exemple, l'emploi du modal marque l'ajustement de l'énonciateur à la situation, ajustement qui s'effectue en acceptant la volonté de l'interlocuteur.

(1) KAREN: Hold on, Jonah. (into phone) L'hopital? Le medecin est là? Le docteur....Oui. This is an emergency.....oui...how long?.....Yes, I'll wait. J'attends....

She waits. The piano trails off as the lights fade. (J. Fleming, Burnt Piano)

Le fondement de la visée peut être plus abstrait lorsqu'il émane d'un raisonnement. Il y a alors ajustement à un principe général :

(2) PETE: What's the boy doing?

KAREN: He works for Godot

PETE: Not that boy. Your boy. Our Jonah?

KAREN: Oh. Asleep.

PETE: No nightmares.

KAREN: Shh. He'll hear you. (J. Fleming, Burnt Piano)

En (2), en l'absence de proposition explicite en if, l'interjection shh nous permet de mettre en rapport he'll hear you avec un implicite if you go on talking like this. He'll hear you est lié à $\mathrm{T}_{0}$ par cette condition implicite. On sait que l'on se fait entendre lorsque l'on fait du bruit, et cette connaissance sert à mettre en garde l'interlocuteur dans cette situation spécifique.

Dans le second type d'emploi, le but n'est pas de prédire une occurrence future. Il s'agit plutôt d'expliquer une situation ou une attitude. On peut ainsi mettre en avant un principe général dans une situation donnée :

2 Quirk \& al. (1985 : 228) considèrent que historiquement et sémantiquement, ' $l l$ et ' $d$ sont les contractions, respectivement, de will et de would et non de shall et should. Ils montrent notamment que la substitution de should par ' $d$ et de shall par 'll n'est pas possible dans des contextes où seuls shall et should sont acceptables. Huddleston \& Pullum (2002 : 91) montrent également que ' $l l$ est la forme clitique de will, forme réduite phonologiquement et non syntaxiquement. 
(3) KAREN: (To herself) Oh, God... (to him) Do you believe in any kind of Fate? SAM: The Irish will believe in anything, provided it doesn't require the oppressive intervention of logic. (J. Fleming, Burnt Piano)

On peut remarquer ici qu'il manque une étape dans l'argumentation : il n'y a pas de réponse à la question personnelle qui est posée. Au lieu de répondre 'I do' à titre personnel, le locuteur avance un principe général permettant de justifier sa bizarrerie $^{3}$ en situation, bizarrerie qu'il met au compte de sa nationalité. La situation vient exemplifier un trait de caractère typique des irlandais, ce que l'on peut traduire en français par «quand on est irlandais, on croit à n'importe quoi... $»^{4}$. Comme dans les exemples précédents, c'est donc bien l'ajustement à un principe général - ou ironiquement présenté comme tel - qui motive l'emploi de will, mais dans le but de justifier un comportement en situation et non pour prédire une occurrence à venir.

\section{WILL BE -ING}

\subsection{Prédiction reposant sur la conformité à un préconstruit}

Qu'est-ce qui change avec l'ajout de be-ing? Depuis Coates (1983), il est établi que l'aspect associé aux modaux est le plus sûr moyen de désactiver toute valeur radicale au profit de la valeur épistémique. Il en découle une modification de la relation intersubjective, puisque la valeur de volonté, dans le cas de will, se trouve désactivée. La différence de sens est nette dans les questions à la $2^{\text {ème }}$ personne :

(4) 'Will you stay, or won't you stay, Horace?' Narcissa said. 'I think I'll stay,' Horace said. (W. Faulkner, Sanctuary)

(5) 'Will you be going away for Christmas, Miss Parchman?' said Audrey, who prided herself, as Jaqueline did, on having a fund of small-talk suitable for persons in every rank of life. (R. Rendell, A Judgement in Stone)

En (4), l'interlocuteur est interrogé sur sa décision en $\mathrm{T}_{0}$, tandis qu'en (5), la question est une simple demande d'information concernant un programme déjà arrêté. Le rôle de l'aspect be-ing ici n'est pas de renvoyer à un procès en cours dans l'avenir, mais de marquer le choix de "go away » comme préconstruit en $\mathrm{T}_{0}$. Comme le fait remarquer Delmas (1998: 171), la valeur aspectuelle de being se trouve neutralisée sous l'influence du modal.

Selon Coates (1983: 180), la forme be-ing associée à will permet au locuteur de garder une certaine neutralité en posant une question concernant un choix

\footnotetext{
${ }^{3}$ Nous reprenons ce terme de Larreya \& Rivière (2005 : 114).

4 Nous citons ici la traduction par J.-P. Richard de Fleming, Justin, Le piano brûlé. Carnières : Editions Lansman, 2004.
} 
indépendant de la situation d'énonciation et indépendant de lui. En formulant ainsi sa question, le locuteur ne peut exercer une quelconque pression sur l'interlocuteur, alors que will seul peut parfaitement renvoyer à une valeur jugée souhaitable par le locuteur.

En accord avec Coates, nous estimons qu'avec will, le sujet you est posé comme origine agentive du procès et comme instance de prise en charge, dont la réponse a valeur d'engagement (cf. (4) : I think I'll stay). Au contraire, avec will + be-ing, la réponse consiste à énoncer ce qui est prévu concernant un sujet privé de toute propriété agentive, sans que l'énonciateur s'engage.

Mais il faut aller plus loin, car l'emploi de be -ing dépasse largement les cas où le verbe est compatible avec l'agentivité :

(6) Will Madame be requiring a room ? No, says Mimi, thoughtfully; that will not be necessary. (A. Brookner, Family and Friends)

Il serait inexact de dire que la forme be-ing annule ici la volonté, puisque celle-ci est de toute façon absente avec 'require'. Par contre, la question concerne une décision censée être déjà prise préalablement à l'énonciation, ce qui permet au locuteur de ne pas avoir l'air d'exercer une quelconque pression sur l'interlocuteur. Notre hypothèse, c'est que l'énonciation devient médiate, au sens où il n'y a plus de relation directe avec la situation ou l'interlocuteur.

Alors qu'avec will, il y a un ajustement de l'énonciateur à la situation en fonction de sa connaissance du sujet, be-ing associé à will bloque cet ajustement en présentant la prédication comme préconstruite. On peut alors se demander sur quoi se fonde la prédiction. Leech (1971: 62-64) appelle cet emploi "future as a matter of course" et le caractérise ainsi : " this will happen in the natural course of events ». Quirk et al. (1985:216) illustrent cet emploi avec l'exemple suivant que nous leur empruntons :

\section{(7) We'll be flying at 30000 feet.}

Le procès est présenté comme conforme à un protocole aérien et à des prévisions, et non comme résultant d'un choix personnel en réaction à une intempérie ou à un problème technique - auquel cas on aurait will. Cette apparente neutralité explique que l'on ait de plus en plus recours à will + be-ing dans les informations. Le locuteur présente les événements à venir comme attendus, conformes à une norme, et il explicite ainsi que son rôle se limite à relayer une information dont il n'assume pas la responsabilité, ce qui est courant à la radio ou à la télévision :

(8) Liz Savage will be talking about Princess Anne's wedding on Good Morning With Anne \& Nick today at noon. (BNC)

Il nous semble important d'insister sur le fait que cette neutralité n'est qu'apparente. S'il y a bien neutralisation de la relation entre le sujet et le prédicat, c'est au profit d'un jugement de conformité qui revient à l'énonciateur. 
Ce jugement peut servir à masquer toute aspérité dans les événements à venir en les posant systématiquement comme conformes, ce qui n'est peut-être pas aussi neutre que cela vis-à-vis du co-énonciateur, qui n'a aucun moyen de revenir sur une relation préconstruite. Sous couvert de politesse et de tact, l'emploi de cette forme dans une lettre de licenciement, par exemple, présente la décision de mettre fin au contrat de l'employé comme déjà prise et donc inéluctable, incontestable par l'allocutaire et indépendante du locuteur qui se pose comme un simple messager et non comme un agent responsable :

(9) I regret to now inform you that we will be terminating all our contracts with you as of Monday $22^{\text {nd }}$ of July 1991. (ICE-GB, exemple emprunté à Smith 2003 : 717)

La pseudo-neutralité peut ainsi déboucher sur une forme de manipulation où le co-énonciateur n'a plus qu'à accepter ce qui lui est présenté comme allant de soi, sans qu'une quelconque origine de prise en charge soit récupérable dans le contexte.

\subsection{Surprise et appréciation}

Le préconstruit peut être mis en jeu différemment, non plus pour prédire ou rapporter une occurrence future sans prendre en charge sa préconstruction, mais pour porter une appréciation sur la situation. Dans ce cas, on trouve souvent la forme will + be-ing associée à next, one of these days, in a minute... :

(10) 'A garment, you little thief. Mamzer! I'm sorry for your father. Some heir he's got! Some Kaddish! Ham and pork you'll be eating, before his body is in the grave.' (S. Bellow, Herzog)

(11) 'So you mean you haven't read them?' she says, sticking determinedly to the subject of the documents. 'You'll be telling me in a minute you haven't had time. 'Of course I've read them.' (J. Le Carré, The Constant Gardener)

A la différence des cas précédents, la situation est ici le point de départ d'un jugement appréciatif. Confronté de façon directe à une situation qui est surprenante ou contraire à sa norme, l'énonciateur ne vise pas une occurrence future, mais cherche plutôt à dénoncer le cours des événements et à mettre en garde l'interlocuteur contre un type de comportement.

On constate que dans tous les cas, on renvoie à un processus présenté comme indiscutable parce que conforme à un préconstruit. On court-circuite ainsi le rapport à la situation d'énonciation, à laquelle il n'est plus possible de s'ajuster. De façon apparemment paradoxale, ce mode d'énonciation peut être mis en jeu soit pour rapporter en s'effaçant en apparence, soit au contraire pour évaluer ce qui est perçu de façon directe. En fait, dans les deux cas, on a un rapport à l'altérité qui permet d'unifier ces différents effets de sens. En (10) et (11), le cours des événements mène à la valeur $p$ de 'you'll be eating pork' et 'you'll be telling me in a minute you haven't had time'. Or c'est la valeur p' qui est 
attendue par l'énonciateur, d'où l'appréciation défavorable. Dans les exemples précédents, ce n'est pas l'altérité de la valeur du prédicat qui est en question, mais l'altérité de l'origine de prise en charge, d'où l'apparente neutralité et la distanciation.

\section{BE GOING TO}

\section{1. Préconstruction et lien à la situation d'énonciation}

Comme will be -ing, la périphrase be going to, classée parmi les semi-modaux de l'anglais, contient le marqueur aspectuel be -ing et marque également une préconstruction. La préconstruction n'est cependant pas du même type : avec be going to, l'énonciateur, qui présente l'événement à venir comme déjà prévu, prend en charge la prédiction.

Notre hypothèse d'une valeur préconstruite prise en charge par l'énonciateur en $\mathrm{T}_{0}$ doit être précisée, dans la mesure où de nombreuses études se proposant de comparer will et be going to mettent souvent en avant un même critère, d'ordre sémantique: avec be going to, l'événement à venir est vu comme intrinsèquement lié au présent de l'énonciation, alors que will permet de renvoyer à un événement à venir de façon plus «neutre», détachée du présent de l'énonciation. Be going to est ainsi décrit en termes de «present orientation » (McIntosh 1966 : 105) ou de « current orientation » (Palmer 1979 : 121).

Les deux exemples suivants montrent qu'une caractérisation en termes de « lien avec le présent » reste trop floue :

(12) 'Ella, if you don't shut up I'm going to rip your tongue out of your throat.' (R. Cusk, Arlington Park)

(13) It isn't just instinct which tells me he isn't going to like the news about my friendship with the unconventional Baroness. (O. Wynd, The Ginger Tree)

Dans ces énoncés, c'est effectivement un élément présent dans la situation qui permet à l'énonciateur de faire une prédiction : en (12), il s'agit de la décision prise par le locuteur; en (13), c'est la réaction du personnage masculin, précédemment observée par la locutrice, qui permet à celle-ci d'inférer qu'il n'appréciera pas sa nouvelle amitié avec une baronne peu conventionnelle. Il y a donc bien lien ou ancrage situationnel. Mais il nous semble que ce n'est pas tant le fait qu'il y ait un lien avec le présent qui pourrait rendre compte de l'utilisation de be going to que le caractère déjà acquis de l'événement à venir : la décision est déjà prise en (12), tous les signes ${ }^{5}$ montrent que l'événement aura lieu en (13). Be going to associe donc préconstruction et prise en charge en $\mathrm{T}_{0}$.

On retrouve à travers nos deux exemples les deux grandes valeurs de be going to : valeur radicale de décision et valeur épistémique d'inférence, qui constitue

${ }^{5}$ Brisard (2001) fait ainsi l'hypothèse que be going to est lié à la notion de signe, alors que will serait lié à la notion de prémisse empruntée à la logique. 
selon Guentchéva (1996 : 15-16) une des valeurs privilégiées de la catégorie du médiatif dans les langues où cette catégorie grammaticale existe.

\section{2. Décision et inférence}

Qu'il s'agisse de décision ou d'inférence, la préconstruction qui sous-tend be going to permet, sur le plan intersubjectif, d'anticiper toute critique ou discussion de la part de l'interlocuteur, et d'y couper court. Néanmoins, il ne s'agit pas, comme avec will be -ing, de s'abstraire du rapport interlocutif, mais plutôt de s'assurer de la maîtrise de ce rapport.

Ainsi, dans sa valeur radicale de décision, be going to associé à un sujet de première personne sert à bloquer toute remise en cause éventuelle de la décision par l'interlocuteur. C'est bien le cas dans l'exemple (12) cité précédemment: l'emploi de be going to permet à la mère de menacer plus efficacement son enfant qui ne cesse de pleurer. A la deuxième personne, il ne s'agit plus de la décision du référent du sujet syntaxique mais du locuteur (du moins dans les énoncés affirmatifs). Le caractère entériné de la décision permet alors l'émergence d'une nuance injonctive :

(14) 'I haven't finished with you, boy!'

'Get out of the way,' said Harry quietly.

'You're going to stay here and explain how my son -' (J. K. Rowling, Harry Potter and the Order of the Phoenix)

Lorsqu'il y a inférence, be going to permet à l'énonciateur d'asseoir la légitimité de son propos en ne faisant pas reposer sa prédiction sur une simple intuition ou croyance : la prédiction est fondée sur des données situationnelles présentées comme objectives. Ainsi, dans l'énoncé (13), la locutrice oppose très clairement son instinct aux preuves ou signes à l'origine de son inférence. Cela permet de rendre légitimes et de justifier aux yeux des interlocuteurs les jugements exprimés à propos de l'avenir. Dans d'autres exemples, l'inférence peut servir à tenter de rassurer l'interlocuteur :

(15) She spoke in the voice she used to calm desperate parents; slow, light, airy rather than grave. 'It's going to be fine, perfectly fine.' (I. McEwan, Amsterdam)

On peut considérer qu'il y a «médiation » dans la mesure où c'est via les données situationnelles que s'effectue la prédiction, mais celle-ci est forcément associée à une prise en charge énonciative en $T_{0}$ : c'est l'énonciateur qui a fait de ces données des indices, comme le montre tells me dans l'exemple (13) plus haut.

\section{CONCLUSION}

Alors que will, marqueur d'ajustement, établit un rapport direct avec la situation d'énonciation, les deux formes aspectuo-modales will be -ing et be going to sont liées à la reprise d'un préconstruit. 
Avec will be -ing, la préconstruction permet à l'énonciateur de ne pas prendre en charge la relation prédicative : il fait le choix délibéré d'une énonciation "médiate», en ne se présentant pas comme étant à l'origine de cette préconstruction. Will be -ing apparaît ainsi comme le marqueur le plus "neutre » de renvoi à l'avenir en anglais contemporain : l'aspect be -ing associé au modal will aboutit à une neutralisation de la valeur radicale et permet de présenter le procès à venir comme déjà prévu indépendamment de l'énonciateur. Nous avons cependant montré que will be -ing peut aussi être utilisé dans le cadre d'une stratégie intersubjective pour apprécier défavorablement le cours des événements.

Avec be going to, l'énonciateur se présente bien, au contraire, comme l'instance à l'origine de la préconstruction: le caractère préconstruit de la décision et de l'inférence à partir de données situationnelles ne conduit pas à une absence de prise en charge, même si parfois, dans le cas de l'inférence, l'appui sur ces données peut donner une impression d'objectivité.

Ces formes aspectuo-modales font donc intervenir, à des degrés divers, la subjectivité de l'énonciateur: la référence à l'avenir en anglais est nécessairement liée aux représentations de l'énonciateur et semble de plus en plus relever d'une énonciation médiatisée, comme tend à le montrer l'augmentation de will be -ing et de be going to en anglais contemporain.

\section{BIBLIOGRAPHIE}

Berglund Y. 1997, Future in Present-day English: Corpus-based evidence on the rivalry of expressions, ICAME Journal 21. 7-20.

Berglund Y. 2000, Utilising Present-day English corpora: A case study concerning expressions of future, ICAME Journal 24. 25-63.

Brisard F. 2001, Be going to: an exercise in grounding, Journal of Linguistics 37 (2). 251-285

Celle A. \& Smith, N., (à paraître) From aspect to pragmatics: will be -ing and shall be -ing, English Language and Linguistics.

Coates J. 1983, The semantics of the modal auxiliaries, London \& Canberra, Croom Helm.

Delmas C. 1998, Futurité : temps et strates en anglais, in A. Borillo, C. Vetters \& M. Vuillaume (ed), Variations sur la référence verbale, Amsterdam, Atlanta, Rodopi, p. 163-175.

Guentcheva Z. 1996, Introduction, in Z. Guentchéva (ed), L'énonciation médiatisée, Paris, Louvain, Peeters.

Huddleston R. \& Pullum G. 2002, The Cambridge Grammar of the English Language, Cambridge, Cambridge University Press.

Larreya P. et Rivière C., 2005, Grammaire explicative de l'anglais, Paris, Longman

Leech G. 1971, Meaning and the English Verb, London, Longman.

Mair C. 1997, The spread of the going to future in written English. A corpusbased investigation into language change in progress, in R. Hickey \& S. Puppel (ed), Language History and Linguistic Modelling. A Festschrift for Jacek Fisiak on his sixtieth birthday. Berlin, New York, Mouton de Gruyter, p. 1537-1543. 
McIntosh A. 1966, Predictive Statements, in C.E. Bazell, J.C. Catford, M.A.K. Halliday \& R.H. Robbins (ed), In Memory of J.R. Firth, London, Longmans, Green \& Co. Ltd, p. 303-320.

Nesselhauf N. 2007, The spread of the progressive and its 'future' use, English Language and Linguistics 11-1 : 191-207.

Palmer F.R. 1979, Modality and the English Modals, London, Longman.

Quirk R., Greenbaum S., Leech G. \& Svartvik J., 1985, A comprehensive grammar of the English language, New York, Longman.

Smith N. 2003, A quirky progressive? A corpus-based exploration of the will + be + -ing construction in recent and present day British English, in D. Archer, P. Rayson, A. Wilson \& A. McEnery (ed), Proceedings of the Corpus Linguistics 2003 Conference, Lancaster, Lancaster University, UCREL Technical Paper n¹6., p. 714-723. 